\title{
Toma de decisiones y consideraciones éticas en el soporte ventilatorio en áreas críticas hospitalarias en el contexto de pandemia COVID.19
}

\section{Decision-making and ethical considerations in ventilatory support in critical hospital areas in the context of the COVID-19 pandemic}

José Amado 1,a , Virginia Garaycochea, ${ }^{2, \mathrm{~b}}$, José Portugal $^{3, \mathrm{c}}$, Carolina Gonzales ${ }^{3, \mathrm{~d}}$, Liliana Cieza ${ }^{3, \mathrm{e}}$

\begin{abstract}
RESUMEN
Objetivo. Los recursos hospitalarios resultan insuficientes ante la demanda de pacientes graves con COVID-19. La adecuada gestión de recursos es esencial para brindar la mejor atención posible. Se revisaron criterios que ayuden a tomar decisiones adecuadas y oportunas, siguiendo principios éticos. La priorización del soporte ventilatorio invasivo debe hacerse de manera transparente y objetiva, evaluando integralmente al paciente y basado en criterios objetivos como escalas de pronóstico, ciclo de vida o compromiso clínico. El tratamiento sintomático (incluyendo cuidados paliativos) es indispensable en esta pandemia y la comunicación con el paciente o la familia permiten humanizar la atención del personal de salud.
\end{abstract}

Palabras Clave: Infecciones por Coronavirus; Betacoronavirus; Síndrome respiratorio agudo grave; Ventiladores mecánicos; Enfermedad crítica; Ética clínica; Manejo de Atención al Paciente (Fuente: DeCS-BIREME).

\begin{abstract}
Objetive. Hospital resources are insufficient given the demand for seriously ill patients with COVID-19. Proper resource management is essential to provide the best possible care. Criteria to help make appropriate and timely decisions were reviewed, following ethical principles. The prioritization of invasive ventilatory support must be done in a transparent and objective manner, comprehensively evaluating the patient and based on objective criteria such as prognostic scales, life cycle or clinical commitment. Symptomatic treatment (including palliative care) is essential in this pandemic and communication with the patient or family makes it possible to humanize the care of health personnel.
\end{abstract}

Keywords: Coronavirus Infections; Betacoronavirus; Severe Acute Respiratory Syndrome; Ventilators, Mechanical; Critical Illness; Ethics, Clinical; Patient Care Management (Source: DeCS-BIREME).

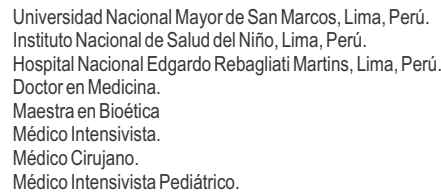

\section{INTRODUCCIÓN}

\section{GENERALIDADES SOBRE COVID-19}

COVID-19 (del inglés Coronavirus Disease 2019) es una enfermedad causada por el Virus del Síndrome Respiratorio Agudo Severo 2 (SARS-CoV-2) un virus RNA de la familia de los coronavirus. Se inició en diciembre de 2019 en Hubei (China) con la presentación de casos de neumonía grave asociados a un mercado de la ciudad. Postulándose un origen zoonótico, vinculando al pangolín como su huésped intermediario y al hombre su huésped final. La principal vía de transmisión es por aerosoles en espacios cerrados, con una alta capacidad de propagación, siendo susceptible toda la población ${ }^{(1)}$.

El virus invade las células del huésped produciendo lesiones pulmonares severas, exfoliación del epitelio bronquial, pérdida de cilios y daño alveolar difuso con formación de membrana hialina y posterior fibrosis. Se han identificados virus en linfocitos, monocitos y tejido linfoide del aparato digestivo, riñones y sistema nervioso central. Se ha descrito una gran activación y agregación plaquetaria con depósito de fibrina en el endotelio vascular que promueven fenómenos trombóticos en vasos pequeños periféricos ${ }^{(1,2)}$. 
Tiene un periodo de incubación promedio de 5-6 días (hasta 14 días). Los síntomas iniciales son fiebre moderada, tos, trastornos del gusto y el olfato, y con menos frecuencia síntomas digestivos (diarrea). En el $80 \%$ de los casos se desarrolla un cuadro leve a moderado similar a una gripe y se resuelve en 10 a 14 días. En los casos graves (15\%) o muy graves $(5 \%)$, después de 7 a 10 días se presenta disnea, aumento de frecuencia respiratoria e insuficiencia respiratoria severa. La tomografía muestra opacidades pulmonares en vidrio deslustrado de distribución en empedrado periférico bilateral y consolidados lobares con bronquiectasias. Desarrollándose síndrome de insuficiencia respiratoria aguda que requiere cuidados intensivos y soporte ventilatorio avanzado ${ }^{(1,2)}$.

Se han ensayado varios medicamentos, pero hasta la fecha ningún tratamiento específico ha demostrado ser efectivo. Los casos leves o moderados rara vez se hospitalizan. Los casos muy graves requieren soporte ventilatorio invasivo. Desarrollan insuficiencia respiratoria hipoxémica, siendo la terapia con oxígeno suplementario parte fundamental del manejo. Sin embargo, cuando el compromiso pulmonar es muy significativo, no son suficientes los dispositivos comunes (cánula binasal o máscara con reservorio) y se hacen necesarios otros tratamientos como la ventilación no invasiva (incluyendo las modalidades de cánula de alto flujo o snorkel) y ventilación mecánica invasiva. La oxigenación con circulación extracorpórea (EMCO) también se han utilizado en casos graves de COVID-19 $19^{(2,3)}$.

El COVID-19 en pediatría ha tenido aspectos diferentes con respecto a los adultos, los primeros meses de pandemia sólo se presentaba enfermedad leve y los pocos niños que requirieron cuidados intensivos tenían en su mayoría comorbilidades avanzadas o eran menores de 1 año. Sin embargo, se demostró que los niños tienen mayores cargas virales incluso siendo asintomáticos por lo que pueden transmitir la enfermedad con mayor facilidad ${ }^{(4,5)}$. Desde finales de abril 2020, se reportan casos de síndrome parecido al Síndrome de Kawasaki con shock asociado, semanas después de infección por SARS-COV-2, definiéndose a la fecha criterios de síndrome inflamatorio multisistémico pediátrico asociado a SARS-CoV-2. Este síndrome es más frecuente en mayores de 5 años, sin comorbilidad previa, con importante afectación cardíaca y alta necesidad de terapia intensiva; presentando buena respuesta a inmunoglobulina y corticoides $^{(4,6)}$.

Hasta el seis de setiembre de 2020 se han confirmado en el mundo 27 millones de infectados por SARS-CoV-2, 889 mil fallecimientos y América es el actual epicentro de la pandemia. SARS-CoV-2 tiene menor tasa de mortalidad que SARS-CoV y MERS-Cov, pero viene infectando a mayor número de pacientes. Su tasa bruta de mortalidad es de 1,4 a $7,0 \%{ }^{(7)}$. En Perú se han reportado 689977 pruebas positivas, 29838 fallecidos, 1500 casos se encontraban en ventilación mecánica correspondiendo al $13,24 \%$ de los 11327 hospitalizados $^{(8)}$.

La COVID-19 ha producido un gran impacto económico y psicológico en el mundo (especialmente en los países con menos recursos para el cuidado de la salud) por el aislamiento social que se ha instaurado para tratar de disminuir su efecto. La estancia hospitalaria es prolongada y la mortalidad muy alta; modificando los sistemas de salud de todo el mundo ${ }^{(2,3)}$. Se han publicado recomendaciones clínicas en pacientes con COVID-19 tratados en áreas no críticas $^{(9)}$, pero sobre toma de decisiones y consideraciones éticas respecto a soporte ventilatorio existen publicaciones con criterios no uniformes $^{(10)}$.

En este contexto, las acciones y decisiones del equipo médico tienen gran importancia en la atención del paciente con necesidad de soporte ventilatorio ${ }^{(11)}$. Esta revisión busca esclarecer pautas, basadas en normas nacionales e internacionales, para guiar el trabajo del médico en áreas críticas con el debido marco bioético que garantice el respeto de los Derechos Humanos en la toma de decisiones, durante esta pandemia.

\section{Metodología de búsqueda bibliográfica}

Se realizó búsqueda bibliográfica en PubMed y Google Scholar, en idiomas inglés y español, hasta el 31 de agosto de 2020, seleccionando los artículos de revisión, guías de práctica clínica y documentos técnicos con acceso a manuscrito completo. Se identificaron 202 publicaciones, 48 publicaciones luego de excluir duplicados, 16 documentos evaluados a texto completo y finalmente siete documentos fueron incluidos (Tabla1).

Términos de búsqueda: (COVID-19 OR SARS-CoV-2) AND ("Síndrome respiratorio agudo grave" OR "ventiladores mecánicos" OR "Enfermedad crítica") AND ("Ética clínica" OR "Manejo de Atención al Paciente").

("COVID-19" [Supplementary Concept] OR "severe acute respiratory syndrome coronavirus 2" [Supplementary Concept]) AND ("Severe Acute Respiratory Syndrome"[Mesh] OR "Ventilators, Mechanical"[Mesh] OR "Critical Illness"[Mesh]) AND ("Ethics, Clinical"[Mesh] OR "Patient Care Management"[Mesh])

En PubMed se encontraron 68 artículos y Google Scholar en español e inglés 100, revisándose además 34 citas adicionales. Las citaciones evaluadas luego de excluir duplicados son 48, encontrándose 16 documentos evaluados a texto completo, siendo 7 los documentos incluidos. 
Tabla 1. Lista de artículos incluidos

\begin{tabular}{|c|c|c|c|}
\hline Autor/Institución & Título & País & $\begin{array}{l}\text { Fecha de } \\
\text { Publicación }\end{array}$ \\
\hline Ballesteros et al / SEMICYUC & $\begin{array}{l}\text { Recomendaciones de "hacer" y "no hacer" en el tratamiento de los pacientes críticos ante la pandemia por } \\
\text { coronavirus causante de COVID-19 de los Grupos de Trabajo de la Sociedad Española de Medicina Intensiva, Crítica y } \\
\text { Unidades Coronarias }\end{array}$ & España & Ago-20 \\
\hline Arévalo-Leal et al & $\begin{array}{l}\text { Problemas éticos en la asignación de recursos escasos y cuidado intensivo en la atención de la pandemia por SARS } \\
\text { CoV-2 }\end{array}$ & Colombia & Jul-20 \\
\hline Leclerc et al & Prioritisation of ICU treatments for critically ill patients in a COVID-19 pandemic with scarce resources & Francia & Jun-20 \\
\hline Colegio Médico del Perú & Orientaciones éticas para la toma de decisiones en la pandemia COVID-19 & Perú & May-20 \\
\hline Valera et al & Orientaciones éticas para la toma de decisiones médicas en el contexto de la pandemia de COVID-19 en Chile & Chile & Abr-20 \\
\hline Daugherty et al & Too Many Patients...A Framework to Guide Statewide Allocation of Scarce Mechanical Ventilation During Disasters & EEUU & Abr-20 \\
\hline World Health Organization & Clinical management of severe acute respiratory infection when novel coronavirus ( $\mathrm{nCoV}$ ) infection is suspected & Suiza & Ene-20 \\
\hline
\end{tabular}

\section{ASPECTOS ÉTICOS EN LA PANDEMIA}

La emergencia sanitaria que estamos viviendo debido a COVID-19, ha conmocionado todo nuestro sistema de salud, especialmente los servicios de emergencia y unidades de cuidados intensivos de los hospitales los cuales se han visto rebasados, no solo por la cantidad de pacientes (con sobredemanda pre-existente), sino por la gran cantidad de fallecimientos no presentadas hace siglos ${ }^{(11-13)}$.

Las primeras semanas de marzo (etapa inicial de la pandemia) las salas de emergencia estaban con menos del $50 \%$ de su capacidad; sin embargo, las siguientes semanas, los pacientes afectados por COVID-19 acudieron masivamente al servicio de emergencia, ocupando todos los ambientes destinados para tal fin, se formaban colas de pacientes esperando atención y hospitalización, muchos de ellos en estado crítico, ocurriendo fallecimientos incluso en la vía pública ${ }^{(13-15)}$.

La atención médica individual reconoce la dignidad de la persona humana y se basa en los principios éticos de no maleficencia, justicia, beneficencia y autonomía. En tiempos de pandemia la bioética se enfoca en la población desde la mirada de la salud pública, buscando beneficiar al mayor número de personas, priorizándose los principios de justicia y no maleficencia para la toma de decisiones, de acuerdo a los recursos disponibles y estableciendo prioridades de atención ${ }^{(11,14,16)}$.

En este marco, las autoridades sanitarias tienen la obligación ética de planificar, proteger y guiar el funcionamiento de la institución, haciendo una justa distribución de los recursos, debiendo adaptarse a los constantes cambios en relación a la pandemia por COVID-19, siendo necesarias acciones de coordinación, organización e impulso al trabajo interdisciplinario. La Organización Mundial de la Salud recomienda establecer criterios de priorización transparentes para salvar la mayor cantidad de vidas, tratar a todas las personas equitativamente (las que presentan COVID-19 y también las que presentan otras enfermedades $)^{(11,17)}$.

El profesional de salud debe conocer las recomendaciones y documentos de consenso de las sociedades científicas locales o de países donde ya han respondido a la pandemia. Las decisiones clínicas y realización de procedimientos deben contar con el consentimiento informado del paciente o sus familiares. Los tratamientos de uso compasivo (offlabel) han de ser administrados informando al paciente o sus representantes de su falta de evidencia actual, probables efectos secundarios y posibilidades de éxito que justifican la recomendación clínica en situaciones de epidemia ${ }^{(16)}$. El respeto de las decisiones del paciente y el trato adecuado a la familia son factores fundamentales, de acuerdo con el principio de autonomía de la bioética ${ }^{(18-20)}$.

Los profesionales de salud no abandonarán a su paciente en situación de aislamiento por su enfermedad infecciosa, lo atenderán con las medidas de protección indicadas todas las veces que sea precisa su atención, explicándoles la justificación de restricción de visitas y atendiendo sus necesidades médicas, psicológicas, espirituales y brindando los cuidados necesarios. Se favorecerá el acompañamiento familiar cuando la situación epidemiológica lo permita y en las condiciones de aislamiento protector que esté recomendado ${ }^{(16)}$.

\section{INDICACIÓN DE SOPORTE VENTILATORIO INVASIVO EN COVID-19}

El creciente número de contagios y la gran demanda de camas en unidades críticas para tratar a los pacientes con insuficiencia respiratoria que requieren de soporte ventilatorio y hemodinámico ha sobrepasado la capacidad instalada de los hospitales, lo que ha motivado que los médicos de emergencia y cuidados intensivos tengan que decidir que paciente debe ingresar a $\mathrm{UCl}$ para brindarle soporte de vida. Esta decisión está basada en criterios médicos y los 
principios bioéticos que norman nuestra profesión claramente expresados por el CMP en el documento, Principios éticos en situaciones de Emergencia Sanitaria ${ }^{(11)}$.

En la fase grave o crítica de la COVID-19 se requiere soporte ventilatorio, aproximadamente en el $5 \%$ de los $\operatorname{casos}^{(1,2)}$. La decisión de iniciar soporte intensivo incluye asegurar la vía aérea (intubación orotroqueal) y respiración asistida (ventilación mecánica). En COVID_19 se han definidos criterios para iniciar y no iniciar soporte ventilatorio (Tabla 2$)^{(21,22)}$.

Tabla 2. Indicaciones y contraindicaciones para soporte ventilatorio invasivo en pacientes con COVID-19 grave.

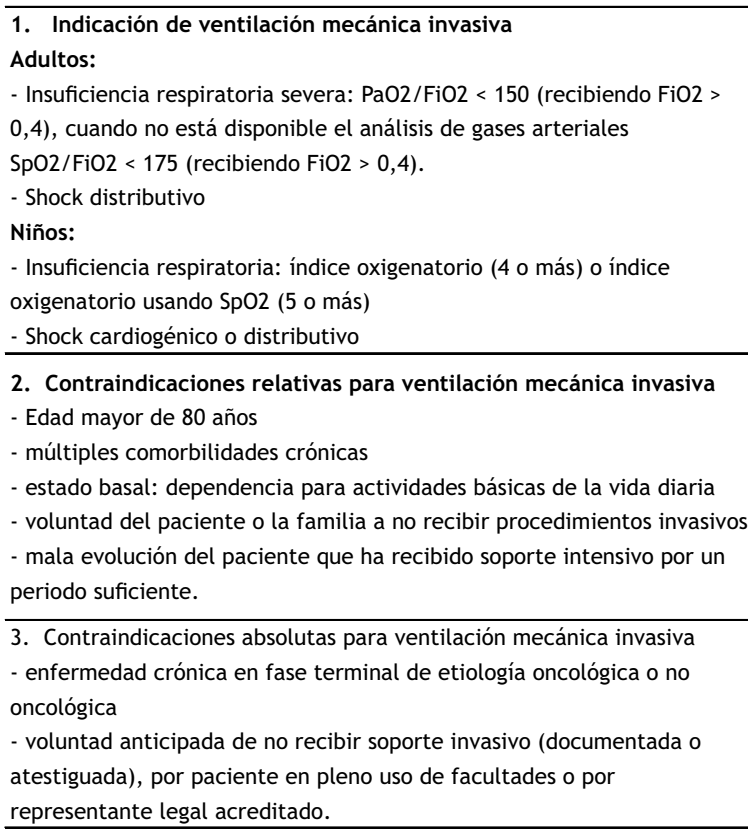

Es necesario definir pautas para los pacientes que requieren soporte ventilatorio en COVID-19 (intubación orotraqueal e ingreso a ventilación mecánica). Este proceso no está exento de dilemas éticos, pero son situaciones reales, frecuentes en pandemias y se deben afrontar basado en criterios objetivos y en el momento adecuado. Se busca salvar la mayor cantidad de vidas, brindando soporte intensivo a los pacientes con mayor esperanza y calidad de vida después del tratamiento ${ }^{(11)}$.

\section{DECISIÓN DE PRIORIZAR SOPORTE VENTILATORIO INVASIVO EN COVID-19}

Cuando los recursos resulten insuficientes, debe realizarse un triaje (selección) en la población que requiere algún tratamiento o procedimiento, a fin de estratificar en base a criterios clínicos de respuesta, recuperabilidad, funcionalidad, y otros, inscritos en un marco ético sólido, donde la justicia distributiva, busca maximizar el beneficio global a aquellas personas con mejores posibilidades de respuesta al mismo. No se pretende decidir entre la vida y la muerte, sino que el paciente reciba los cuidados que necesita, cuando los necesita y en la medida disponible de la forma más justa $^{(16,22)}$.

Una recomendación de expertos en EEUU plantea estrategias para asignación de ventiladores mecánicos basadas en el pronóstico de vida (a corto y largo plazo) y otras consideraciones como edad ${ }^{(23)}$; a esta propuesta se agrega estancia estimada, hallazgos radiológicos o ser profesional de salud (Tabla 3). La evaluación de cada caso asigna un puntaje y el que tenga menor puntuación tendrá mayor prioridad en caso no se disponga ventiladores para todos. Siempre debemos tener presente la evaluación al paciente en forma integral y no solo la enfermedad ${ }^{(16,22,24,25)}$.

La evaluación de los pacientes que están en ventilación mecánica debe ser continua en el tiempo, si las condiciones clínicas no mejoran o se deterioran a pesar del tratamiento intensivo después de un periodo determinado (48 o 72 horas), se puede considerar el retiro del soporte ventilatorio e iniciar terapia paliativa ${ }^{(10,22)}$.

Tabla 3. Estrategias propuestas para asignar ventilador mecánico a pacientes con COVID-19(16,22-24).

\begin{tabular}{|c|c|c|c|c|c|}
\hline \multirow{2}{*}{ Principio } & \multirow{2}{*}{ Especificación } & \multicolumn{4}{|c|}{ Sistema de puntuación } \\
\hline & & 1 & 2 & 3 & 4 \\
\hline \multirow{2}{*}{ Pronostico a corto plazo } & Adultos: SOFA & $<9$ & $9-11$ & $12-14$ & $>14$ \\
\hline & Niños: PELOD-2 & $<12$ & $12-13$ & $14-16$ & $>16$ \\
\hline Pronostico a largo plazo & Comorbilidad & & Enfermedad crónica avanzada & Pronóstico de vida $<1$ año & \\
\hline \multicolumn{6}{|l|}{ Otras consideraciones: } \\
\hline Ciclo de vida & Edad (años) & $0-49$ & $50-69$ & $70-84$ & $850+$ \\
\hline Compromiso tomográfico & $\%$ compromiso pulmonar & & & $>80 \%$ & \\
\hline Duración de Ventilación mecánica & Días estimados & $<3$ & $3-7$ & $7-14$ & $>14$ \\
\hline Personal de salud & Personal de salud activo & SI & NO & & \\
\hline
\end{tabular}

SOFA = Sequential Organ Failure Assessment; PELOD-2 = Pediatric Logistic Organ Dysfunction 2 (Ver Tabla 4)

Modificado en base a Daugherty Biddison EL, Faden R, Gwon HS, Mareiniss DP, Regenberg AC, Schoch-Spana M, et al. Too Many Patients. A Framework to Guide Statewide Allocation of Scarce Mechanical Ventilation During Disasters. Chest [Internet]. 2019 Apr;155(4):848-54. 


\section{CONTROL DE SINTOMAS EN COVID-19}

Además de buscar instaurar el mejor tratamiento para controlar o disminuir la progresión de la enfermedad, es obligatorio contemplar un enfoque de alivio de síntomas para el gran sufrimiento que produce esta enfermedad ${ }^{(18-20)}$.

Disnea y dolor son síntomas frecuentes en los pacientes con la forma grave de COVID-19 y requieren tratamiento adecuado. Los opioides (principalmente morfina) son los medicamentos de elección y deben ser indicados a la dosis adecuada, considerando también las medidas adicionales para prevenir o controlar sus efectos adversos ${ }^{(10)}$. El delirium se asocia con frecuencia al paciente crítico, debe reconocerse y tratarse precozmente, también requiere un enfoque integral y entre las alternativas farmacológicas se consideran benzodiacepinas, haloperidol, levomepromazina o quetiapina $^{(19)}$.

Los pacientes ingresados a ventilación mecánica requieren sedación, analgesia y relajación muscular por vía endovenosa continua. Midazolam, fentanilo, propofol y vecuronio son los medicamentos más utilizados, pero en tiempos de pandemia no estaban disponibles y debieron usarse alternativas como diazepam, morfina, rocuronio o ketamina. Esta descontinuación del tratamiento empeora su evolución y sufrimiento.

Otra situación (muy frecuentes en hospitales) es la mala evolución del paciente a pesar de todo el soporte, reportándose tasas de mortalidad en $\mathrm{UCl}$ de $49 \%{ }^{(26)}$; 0 peor aún, la gran sintomatología por la falta de medicación o equipos adecuados, cuando la demanda sobrepasa los recursos del sistema de salud.

Los cuidados paliativos constituyen los cuidados brindados por un equipo humano multidisciplinario de acompañamiento y soporte a la persona y su familia que sufren una enfermedad que amenaza o acorta la vida, situación que es evidente en la actual pandemia por COVID-19. Los cuidados paliativos no pretenden adelantar ni retrasar el proceso natural de su muerte y en este contexto son imprescindibles ${ }^{(18-20)}$, pues proporcionan el enfoque de soporte integral a los pacientes y familias que incluye el entorno físico, psicológico, social y espiritual ${ }^{(27)}$.

Otro aspecto a tener en cuenta es la valoración del tratamiento adecuado en el contexto clínico de cada paciente, este debe ser con la indicación precisa, con dosis adecuada de los medicamentos y en el momento oportuno; buscando evaluar el impacto de las medidas en el proceso de enfermedad y además en la calidad de vida de la persona. Debemos evitar caer en indicaciones terapéuticas fútiles que solo estarían prolongando un estado de agonía y con gran sufrimiento (distanasia, obstinación, encarnizamiento o ensañamiento terapéutico); evitando también la eutanasia, al dar medicamentos con el fin de acelerar la muerte del paciente ${ }^{(28)}$. Ambas opciones, el encarnizamiento terapéutico como la eutanasia son éticamente reprochables.

\section{MANEJO DE INFORMACIÓN}

La información al paciente y sus familiares se considera un pilar fundamental en la atención clínica, el titular del derecho a la información es el paciente y en caso de incapacidad para comprender o decidir sobre su estado de salud, su familiar o representante ${ }^{(16)}$. En tiempos de pandemia, la información debe ser clara y oportuna tanto para los pacientes, los familiares y el personal de salud. Se debe asegurar el flujo de información veraz y constante, priorizando la forma virtual para evitar la propagación de la infección. Esto brinda un soporte psicológico y mejora la salud mental ${ }^{(14)}$.

Una mala noticia es una información que cambia drásticamente la perspectiva del paciente de sí mismo en relación con su entorno y el futuro. El equipo de salud que atiende los pacientes COVID-19, o equipos anexos a ellos, deben contar con habilidades en la comunicación de malas noticias como la complicación de la situación de salud, informar de un fallecimiento, que el paciente no es candidato para cuidados intensivos, quien será candidato a sedación paliativa, entre otras; con el fin de humanizar la atención médica, con una propuesta empática a los momentos difíciles por los que pasamos ${ }^{(29)}$.

Debido a la situación de distanciamiento social para evitar la propagación de la COVID-19 incluso el informe del fallecimiento del paciente, se hace necesario realizarlo por vía virtual (llamada telefónica), lo que debe realizarse de la mejor forma posible. Se describen pasos para informar al paciente o familiar en este contexto: preparación de la persona que dará el informe, percepción del estado del receptor, brindar la información, responder a las emociones del receptor y cierre del proceso ${ }^{(29)}$.

Es importante mantener el principio de Confidencialidad, es decir que no podrá ser divulgada información del paciente fuera del ámbito sanitario sin consentimiento del paciente o de su representante. La confidencialidad del paciente debe ser protegida, exceptuando requerimiento de información por temas de salud pública ${ }^{(16)}$.

\section{CONCLUSIONES}

En esta Pandemia COVID-19, debe establecerse la forma más adecuada de gestionar los escasos recursos en salud, a fin de brindar la más óptima atención posible, dar el mejor tratamiento a quien lo requiere, con la oportunidad debida, luego de una adecuada valoración y en el marco de respeto de los derechos humanos. 
La priorización de la atención en áreas críticas, y la asignación para el uso de Ventiladores Mecánicos, deberá hacerse de manera humana, transparente, objetiva, científica y racional, en base a la mejor evidencia posible.

Los Cuidados Paliativos, son un elemento indispensable en la atención de pacientes COVID-19, y debieran ser impulsados y promovidos en los establecimientos de salud, especialmente los que cuenten con unidades críticas.

La comunicación veraz, constante y virtual permite una atención más humanizada, debiendo ser promovida en todos los niveles de atención de salud.

Tabla 4. Descripción de Score SOFAy PELOD-2 A. Score SOFA (Sequential Organ Failure Assessment)

\begin{tabular}{lccccc}
\hline \multicolumn{1}{c}{ Variable } & 0 & 1 & 2 & 3 & 4 \\
\hline PaO2/FiO2 $\mathrm{mmHg}$ & $>400$ & $<400$ & $<300$ & $<200$ & $<100$ \\
Plaquetas $\times 10^{3} / \mathrm{ul}$ & $>150$ & $<150$ & $<100$ & $<50$ & $<20$ \\
Bilirrubina $\mathrm{mg} \%$ & $<1,2$ & $1,2-1,9$ & $2,0-5,9$ & $6,0-11,9$ & $>12$ \\
Hipotensión & NO & PAM $<70$ & Dop $<5$ & Dop $6-15$ o NE & Dop $>15$ o \\
(mmHg) & 15 & $13-14$ & $10-12$ & $6-9$ & $\mathrm{NE}>0,1$ \\
Escala Glasgow & 15 & $<6$ \\
Creatinina mg\% & $<1,2$ & $1,2-1,9$ & $2,0-3,4$ & $3,5-4,9$ & $>5$ \\
\hline
\end{tabular}

PAM: presión arterial media Dop: dopamina NE: norepinefrina Fuente: Adaptado de Singer et al (2016)

Calculadora disponible en línea:

http://www.samiuc.es/sofa-score/

\section{B. PELOD-2 (Pediatric Logistic Organ Dysfunction 2) ${ }^{(31)}$}

\begin{tabular}{ll}
\hline Variable & Puntos según severidad 1 a 6 \\
\hline Neurológico: & $110+; 5-10 ; 3-4$ \\
$\begin{array}{l}\text { Puntaje de Glasgow } \\
\text { Diámetro pupilar }\end{array}$ & Reactivas, no reactivas
\end{tabular}

\begin{tabular}{|c|c|}
\hline Cardiovascular: & \\
\hline lactatemia (mmol/L) & $<5 ; 5-10 ; 11$ o + \\
\hline Presión arterial media $(\mathrm{mmHg})$ & Según grupo etáreo \\
\hline Renal: creatinina (mcmol/L) & Según grupo etáreo \\
\hline Respiratoria: & \\
\hline $\mathrm{PaO} 2(\mathrm{mmHg}) / \mathrm{FiO} 2$ & $610+; 600-$ \\
\hline $\mathrm{PaCO} 2$ (mmHg) & 58 o +; 59-94; $950+$ \\
\hline Ventilación mecánica & $\mathrm{No} ; \mathrm{Si}$ \\
\hline Hematológica: & \\
\hline Recuento de leucocitos $\left(\times 10^{3} \mathrm{~L}\right)$ & $20+;$ menos de 2 \\
\hline Plaquetas $\left(\times 10^{3} / \mathrm{L}\right)$ & 142 o +; 77-141; 76 o - \\
\hline
\end{tabular}

Calculadora disponible en línea

https: / / espnic-online.org/Education/ProfessionalResources/Paediatric-Logistic-Organ-Dysfunction-2Score-Calculator
Conflictos de interés: Los autores niegan conflictos de interés.

Financiamiento: Autofinanciado.

\section{REFERENCIAS BIBLIOGRÁFICAS}

1. Yang Y, Xiao Z, Ye K, He X, Sun B, Qin Z, et al. SARSCoV-2: characteristics and current advances in research. Virol J. 2020;17(1):117.

2. Romano CM, Chebabo A, Levi JE. Past, present, and future of COVID-19: a review. Braz J Med Biol Res. 2020;53(9):e10475.

3. Fierabracci A, Arena A, Rossi P. COVID-19: A review on diagnosis, treatment, and prophylaxis. Int J Mol Sci. 2020;21(14): 5145.

4. LuX, Zhang L, Du H, Zhang J, Li YY, Qu J, et al. SARSCoV-2 Infection in Children. N Engl J Med. 2020;382(17):1663-1665.

5. Shekerdemian LS, Mahmood NR, Wolfe KK, Riggs BJ, Ross CE, McKiernan CA, et al. Characteristics and Outcomes of Children With Coronavirus Disease 2019 (COVID-19) Infection Admitted to US and Canadian Pediatric Intensive Care Units. JAMA Pediatr. 2020;174(9):1-6.

6. Belhadjer Z, Méot M, Bajolle F, Khraiche D, Legendre A, Abakka S, et al. Acute Heart Failure in Multisystem Inflammatory Syndrome in Children in the Context of Global SARS-CoV-2 Pandemic. Circulation. 2020;142(5):429-36.

7. Johns Hopkins University. Coronavirus Resource Center. COVID-19 Map [Internet]. 2020 [Citado el ...... de $\left.\begin{array}{llllll}2 & 0 & 2 & 0\end{array}\right]$. D is pon ible e n: https://coronavirus.jhu.edu/map.html

8. Ministerio de Salud. Sala situacional COVID-19 Perú [Internet]. 2020 [Citado el 06 de setiembre de 2020]. D i s p o n i b l e e $n$ : https://covid19.minsa.gob.pe/sala_situacional.asp

9. Instituto de Evaluación de Tecnologías en Salud e Investigación. Recomendaciones clínicas para cuidados paliativos en pacientes con COVID-19 hospitalizados en áreas no UCI [Internet]. Lima-Perú; 2020. [Citado el ... de setiembre de 2020]. Disponible https://www.researchgate.net/publication/343676 082_Recomendaciones_clinicas_para_cuidados_pali ativos en pacientes con_COVID19 hospitalizados en areas no UCl_en_el Seguro Social_del_Peru_EsSalud.

10. Valera L, Carrasco MA, López $R$, Ramos $P$, von Bernhardi R, Bedregal P, et al. Orientaciones éticas para la toma de decisiones médicas en el contexto de la pandemia de COVID-19 en Chile. Rev Med Chil. 2020;148(3):393-8.

11. Ministerio de Salud. Orientaciones éticas para la toma de decisiones en la pandemia COVID-19 [Internet]. Lima; 2020 [Citado el 06 de setiembre de $\left.\begin{array}{lllll}2 & 0 & 2 & 0\end{array}\right] \quad$ D is pon ible e $n$ : https://cdn.www.gob.pe/uploads/document/file/8 71496/consideraciones-eticas-para-la-toma-dedecisiones-en-los-servicios-de-salud-durante-lapandemia-covid-19-v2.pdf. 
12. Ministerio de Salud. Documento Tecnico: Prevención y Atención de personas afectadas por COVID-19 en el Perù [Internet]. Resolución Ministerial. Lima-Perú; 2020 [Citado el 06 de setiembre de 2020]. p. 1-38. D i s p o n i ble e $n$ : https://cdn.www.gob.pe/uploads/document/file/5 74295/resolucion-ministerial-139-2020-MINSA.PDF.

13. Acosta G, Escobar G, Bernaola G, Alfaro J, Taype W, Marcos C, et al. Caracterización de pacientes con COVID-19 grave atendidos en un hospital de referencia nacional del Perú. Rev Peru Med Exp Salud Publica. 2020;37(2):1-5.

14. Taype W, Amado J.Respuesta del servicio de emergencia de un hospital terciario durante el inicio de la pandemia COVID-19 en el Perú. An Fac Med. 2020;81(1):2-7.

15. Haro AS, Calderón EP. Caracterización epidemiológica de Covid-19 en Ecuador Epidemiological characterization of Covid-19 in Ecuador. InterAm J Med Health 2020;3:e202003020.

16. Sanz MÁB, Hernández-Tejedor A, Estella Á, Rivera JJJ, et al. Recomendaciones de «hacer» $y$ «no hacer» en el tratamiento de los pacientes críticos ante la pandemia por coronavirus causante de COVID-19 de los Grupos de Trabajo de la Sociedad Española de Medicina Intensiva, Crítica y Unidades Coronarias (SEMICYUC). Med Intensiva. 2020;44(6):371-388.

17. World Health Organization (WHO). Ethics and COVID19 : resource allocation and priority-setting [Internet]. World Health Organization. 2020 [Citado el 06 de setiembre de 2020]. Disponible en: https://www.who.int/ethics/publications/ethicsand-covid-19-resource-allocation-and-prioritysetting/en/

18. Ouchi K, George N, Revette AC, Hasdianda MA, Fellion L, Reust A, et al. Empower Seriously Ill Older Adults to Formulate Their Goals for Medical Care in the Emergency Department. J Palliat Med. 2019;22(3):267-73.

19. SECPAL. Orientaciones Sobre El Control Sintomático De Enfermos Graves Afectados Por La Enfermedad Covid 19 Y Que Requieran Atención Paliativa O Se Encuentren Próximos Al Final De La Vida [Internet]. 2020 [Citado el .... de setiembre de 2020]. Disponible en:

https://www.secpal.com/biblioteca_documentoscovid19-1

20. Amado J, Oscanoa T. Definiciones, criterios diagnósticos y valoración de terminalidad en enfermedades crónicas oncológicas y no oncológicas Definitions, diagnostic criteria and terminality assessment of cancer and non- cancer chronic diseases. Horiz Med (Lima)2020; 20(3):e1081

21. World Health Organization. Clinical management of severe acute respiratory infection when novel coronavirus ( $\mathrm{nCoV}$ ) infection is suspected [Internet]. Who. 2020 [Citado el .... de setiembre de 2020]. Disponible en: https://www.who.int/internalpublications-detail/clinical-management-of-severeacute-respiratory-infection-when-novelcoronavirus - (ncov) - infection-is suspected\%0Ahttp://apps.who.int/iris/bitstream/1 0665/178529/1/WHO_MERS_Clinical_15.1_eng.pdf

22. Leclerc T, Donat N, Donat A, Pasquier P, Libert N, Schaeffer E, et al. Prioritisation of ICU treatments for critically ill patients in a COVID-19 pandemic with scarce resources. Anaesth Crit Care Pain Med. 2020;39(3):333-9.

23. Biddison ELD, Faden R, Gwon HS, Mareiniss DP, et al. Too Many Patients...A Framework to Guide Statewide Allocation of Scarce Mechanical Ventilation During Disasters. Chest. 2019;155(4):848-854.

24. Sokol D. Guia ética para toma de decisiones en triaje. 2020.

25. Arévalo-Leal JS, Córdoba AIG, Bustamante BJP, León NA, Niuman AC, Peña CET. Problemas éticos en la asignación de recursos escasos y cuidado intensivo en la atención de la pandemia por SARS CoV-2. Acta Médica Colomb. 202016;45(3):1-8.

26. Phua J, Weng L, Ling L, Egi M, Lim C-M, Divatia JV, et al. Intensive care management of coronavirus disease 2019 (COVID-19): challenges and recommendations. Lancet Respir Med. 2020 May;8(5):506-517.

27. Societat Balear de Cures Palliatives. Recomendaciones de actuación en casos de infección por SARS-CoV-2 en pacientes con necesidades de atención paliativa [Internet]. Documento de consenso entre profesionales de equipos específicos de cuidados paliativos de las Illes Balears. 2020 [Citado el .... de setiembre de 2020]. Disponible en: http://www.caib.es/sites/curespalliatives/ca/cure s p p a l i a t i v e s - c o v i d 19/archivopub.do?ctrl=MCRST3145ZI313934\&id $=313$ $\underline{934}$

28. Reyes GLB, Betancourt G. Adecuación del esfuerzo terapéutico: aspectos éticos y legales relacionados con su práctica. Rev Cuba Med Intensiva y Emergencias. 2017; 16(1):15-27.

29. ASEDP, Fundación de Medicina Paliativa de El Salvador. Guía práctica de cuidados paliativos en situación de pandemia COVID-19 [Internet]. 2020 [Citado el .... de setiembre de 2020]. Disponible en: https: / / cuidadospaliativos.org/blog/wpcontent/uploads/2020/04/Gui\%CC\%81apra\%CC\%81ctica-en-situacio\%CC\%81n-de-PandemiaCOVID-19.pdf

30. Singer M, Deutschman CS, Seymour CW, Shankar-Hari M, Annane D, Bauer M, et al. The Third International Consensus Definitions for Sepsis and Septic Shock (Sepsis-3). JAMA. 2016;315(8):801-810.

31. Dewi W, Christie CD, Wardhana A, Fadhilah R, Pardede SO. Pediatric Logistic Organ Dysfunction-2 (Pelod-2) score as a model for predicting mortality in pediatric burn injury. Ann Burns Fire Disasters. 2019;32(2):135-42.

\section{Correspondencia}

José Percy Amado Tineo.

Dirección: Jr Belisario Flores 238 Dpto 301 - Lince. Lima.

Teléfono: 990452547

Correo: jpamadot@gmail.com

jamadot@unmsm.edu.pe

\section{Revisión de pares}

Recibido: 10/09/2020

Aceptado: 20/11/2020 